\title{
Nouvelles connaissances des formes du phosphate : conséquences sur le cycle du phosphate dans les sédiments des eaux douces peu profondes
}

\author{
H.L. Golterman ${ }^{1}$ \\ C.J. De Groot ${ }^{2}$
}

Mots-clés : phosphate, adsorption, fractionnement, sédiments, diagramme de solubilité.

Abréviations utilisées :

o-P : ortho-phosphate dissous

$\mathrm{Fe}(\mathrm{OOH}) \approx \mathrm{P}:$ phosphate adsorbé au $\mathrm{Fe}(\mathrm{OOH})$

$\mathrm{CaCO}_{3} \approx \mathrm{P}$ : phosphate relié au $\mathrm{CaCO}_{3}$, sous forme d'un mélange de calcaire et de micro-cristaux d'apatite

POAS : phosphate organique particulaire, soluble en milieu acide

POR : phosphate organique particulaire résiduel

$P_{\text {tot }}$ : phosphate total d'un sédiment (mg.g $\left.{ }^{-1}\right)$

La composition chimique des phosphates liés aux sédiments d'eau douce a été analysée et l'adsorption de phosphate sur ces sédiments a été étudiée. Le rôle du $\mathrm{Fe}(\mathrm{OOH})$ dans cette adsorption a été confirmé et peut être décrit par l'isotherme d'adsorption de Freundlich : $\mathbf{P}_{\text {ads }}=$ A. $(0-P)^{B}$

où $\quad P_{\text {ads }}=$ concentration du phosphate adsorbé (par les sédiments ou $\mathrm{Fe}(\mathrm{OOH})$ )

$\mathrm{O}-\mathrm{P}=$ concentration du phosphate dissous

A et $\mathrm{B}=$ constantes

Nous avons calculé les constantes $\mathbf{A}$ et $\mathbf{B}$ par ajustement des courbes par la méthode des moindres carrés ; les deux sont des fonctions du pH. On perd très peu de précision si B est ajusté à 0.33 et $« \mathrm{~A} » \mathrm{a} \mathrm{A}=23626^{*} 10^{(-0.42 \mathrm{pH})}$. L'adsorption peut donc être quantifiée en connaissant la concentration du $\mathrm{Fe}(\mathrm{OOH})$ dans les sédiments et le $\mathrm{pH}$.

Puisque le rôle de $\mathrm{Fe}(\mathrm{OOH})$ est important, la conversion du $\mathrm{Fe}(\mathrm{OOH})$ en $\mathrm{FeS}$ pär exemple, peut avoir une forte influence sur l'adsorption de l'o-P sur les sédiments.

Dans les eaux « dures », la solubilité de l'o-P est en plus limitée par la solubilité de l'apatite, $\mathrm{Ca}_{5}\left(\mathrm{PO}_{4}\right)_{3} . \mathrm{OH}$. Nous avons trouvé que le produit de solubilité est de $10^{-50}$. En combinaison avec l'adsorption quantifiée sur le $\mathrm{Fe}(\mathrm{OOH})$, on peut établir un diagramme de solubilité d'o-P dans l'eau en fonction de la concentration de $\mathrm{Fe}(\mathrm{OOH})$ dans les sédiments, de la concentration de $\mathrm{Ca}^{2}+$ dans l'eau et $\mathrm{du} \mathrm{pH}$. Il est apparu qu'il existait une forte corrélation entre la somme $\left(\mathrm{Fe}(\mathrm{OOH}) \approx \mathbf{P}+\mathrm{CaCO}_{3} \approx \mathrm{P}\right)$ et le $\mathbf{P}$ disponible pour certaines espèces d'algues dans 11 lacs néerlandais.

En outre, on trouve dans les sédiments deux « pools » de phosphate organique, l'un soluble dans les acides ( $\mathrm{HCl}$ ou $\left.\mathrm{H}_{2} \mathrm{SO}_{4}\right)$ et l'autre dans la soude $(\mathrm{NaOH})$. Nous pensons, que pour le premier, il s'agit d'un complexe avec la matière humique, tandis qu'il a été démontré que le deuxième est formé en majorité d'un complexe entre l'inositol hexa phosphate (phytate) et probablement, le $\mathrm{Fe}(\mathrm{OOH})$. Ce complexe peut expliquer pourquoi ce phosphate organique est protégé vis-à-vis des bactéries.

En utilisant le diagramme de solubilité on peut maintenant calculer ce qui se produit si un lac peu profond est chargé en phosphate et pourquoi, si la charge est arrêtée, la situation ne peut s'améliorer que très lentement:

\section{New knowledge of phosphate fractions : consequences for the phosphate cycle in shallow freshwater sediments}

Keywords : phosphate, adsorption, fractionation, sediments, solubility diagramme.

The purpose of this paper is to analyse the different phosphate compounds in sediments of freshwater systems and to quantify the processes by which they are formed and which may lead to the equilibrium between uptake and release.

\footnotetext{
1. Station Biologique de la Tour du Valat, le Sambuc, 13200 Arles, France.

2. « Avec tristesse je dois annoncer la mort soudaine de M. Kees de Groot, survenue le 21 septembre 1994 a Barcelone. Que ce travail soit un monument pour ce jeune chercheur prometteur ; il nous manquera fortement. »
} 
For the analysis a sequential extraction scheme is therefore developed, using chelators (such as NTA and EDTA) to extract first the 2 inorganic phosphates, i.e. the iron and the calcium bound phosphate. $\mathrm{Fe}(\mathrm{OOH}) \approx \mathrm{P}$ is extracted with a Ca-NTA + dithionite solution, (resp. $0.02 \mathrm{M} \mathrm{1}^{-1}$ and $0.05 \mathrm{M}^{-1}$ ) and $\mathrm{CaCO}_{3} \approx \mathrm{P}$ with Na-EDTA $\left(0.05 \mathrm{M}^{-1}\right.$ ). Both extractants are adjusted to the $\mathrm{pH}$ of the sediment to be analysed.

Following these extractions, two pools of org- $\mathrm{P}$ can be identified, using subsequent extractions with $\mathrm{H}_{2} \mathrm{SO}_{4}(0.5 \mathrm{M}$ $1^{-1}$ during $\left.30 \mathrm{~min}\right)$ followed finally by one with $\mathrm{NaOH}\left(2 \mathrm{M} \mathrm{l}^{-1}\right.$, at $90^{\circ} \mathrm{C}$ during $\left.5 \mathrm{~min}\right)$. The advantage of this scheme lies in the fact that the inorganic phosphates are extracted before the organic phosphates, together with the $\mathrm{Fe}(\mathrm{OOH})$ and $\mathrm{CaCO}_{3}$, so that no interactions between these adsorbents and phosphate can disturb the analysis of the organic phosphates after hydrolysis.

The adsorption of ortho-phosphate onto freshwater sediments was studied. The influence of $\mathrm{Fe}(\mathrm{OOH})$ on this adsorption process was confirmed in the laboratory. It was found that this adsorption could be described satisfactory by the Freundlich adsorption isotherm :

$\mathrm{P}_{\mathrm{ads}}=\mathrm{A} \cdot(\mathrm{o}-\mathrm{P})^{\mathrm{B}}$

where $\quad \mathrm{P}_{\mathrm{ads}}=\mathrm{o}-\mathrm{P}$ adsorbed onto the sediments or onto $\mathrm{Fe}(\mathrm{OOH})$ and $\mathrm{A}$ and $\mathrm{B}$ are constants

We have quantified « $A$ » and « $B$ » by least squares fitting ; they are both functions of $\mathrm{pH}$, although for $\mathrm{B}$ only moderately. If, however, $B$ is set at 0.33 little precision is lost, and A can be approximated by : A $=23626 * 10(-0.42$ $\mathrm{pH}$ ). In waters with a low $\mathrm{Ca}^{2+}$ concentration, the adsorption onto the sediments can therefore now be quantified as a function of the annual loading, if the $\mathrm{Fe}(\mathrm{OOH})$ concentration in the sediments and the $\mathrm{pH}$ of the water are known. As $\mathrm{Fe}(\mathrm{OOH})$ plays such an important role in the adsorption process, the conversion, e.g. into $\mathrm{FeS}$ as usually occurs in anoxic sediments of shallow water bodies, will influence the adsorption strongly. The constant « $\mathrm{A}$ » does not only depend on the $\mathrm{pH}$, but on the $\mathrm{Ca}^{2+}$ concentration as well. As yet no formula is available to quantify this influence.

In hard water, the solubility of o-P will also be limited by the solubility product of apatite, $\mathrm{Ca}_{5}\left(\mathrm{PO}_{4}\right)_{3} \mathrm{OH}$. Using published data from the two hard water rivers Rhine and Rhone, we have found an 'apparent' solubility product of $10^{-50}$, not taking into account the influence of the activity coefficient due to ionic strength. With this solubility product the maximal o-P concentration can be calculated as a function of the $\mathrm{Ca}^{2+}$ concentration in the water and the $\mathrm{pH}$. Together with the equilibrium constants of the $\mathrm{Fe}(\mathrm{OOH}) \approx \mathrm{P}$ adsorption complex, we have also calculated the maximal o-P concentration as it depends on the $\mathrm{Fe}(\mathrm{OOH})$ concentration in the sediments and the $\mathrm{pH}$ of the system. Combining these two functions, we have calculated a solubility diagram of the maximal o-P concentration in water in equilibrium with the $\mathrm{Ca}^{2}+$ concentration in the water, the $\mathrm{Fe}(\mathrm{OOH})$ concentration in the sediments and the $\mathrm{pH}$. The solubility of dissolved ortho-phosphate appears to depend only on the $\mathrm{Fe}(\mathrm{OOH})$ concentration in the sediments, the $\mathrm{Ca}^{2+}$ concentration in the water (not the $\mathrm{CaCO}_{3}$ concentration in the sediments) and the $\mathrm{pH}$. In 11 dutch lakes it appeared that the sum of $\left(\mathrm{Fe}(\mathrm{OOH}) \approx \mathrm{P}+\mathrm{CaCO}_{3} \approx \mathrm{P}\right)$ is available for phytoplankton growth.

Furthermore we have demonstrated the existence of two organic phosphate pools in the sediments, the first acid soluble (ASOP) and the second alkaline soluble (ROP). We think that the first pool is a humic acid-phosphate complex although we can only present circumstantial experimental evidence. During the extraction, the phosphate of this pool is hydrolysed and goes into solution as o-P, which makes further identification difficult. The concentration of this pool in the sediments varied between 60 and $225 \mu \mathrm{g} \cdot \mathrm{g}^{-1}$. During desiccation of the sediments of a marsh in the Camargue (Les Garcines), this pool disappeared and became inorganic phosphate.

By using an enzymatic hydrolysis with phytase we could demonstrate that most of the second pool (ROP) was phytate, i.e. inositol hexaphosphate. Its concentration varied between 100 and $300 \mu \mathrm{g} \cdot \mathrm{g}^{-1}$. Experimental evidence suggests that this phosphate is probably complexed with $\mathrm{Fe}(\mathrm{OOH})$. The existence of this complex can explain why this organic phosphate is not available for bacterial mineralisation, is therefore not biodegradable and can consequently accumulate in sediments.

In shallow lakes and marshes receiving an important $\mathrm{P}$ loading, we can now quantify with the solubility diagram what will happen with the phosphate concentrations in water and sediments. It appears that during a first phase, nearly all phosphate will enter the sediments, while the concentration in the water increases only very slowly. Furthermore we can predict what will happen later when the $P$-loading is stopped : depending on the water retention time, the situation will only improve very slowly and it may take even longer than the period of loading to return to the low, initial concentrations. In marshes without water renewal, the concentration will remain constant. The available phosphate may, however, still decline because of an accumulation of non-bioavailable phosphate, e.g. phytate. 


\section{Introduction}

Le phosphate entrant dans un écosystème aquatique est, en grande partie, incorporé dans les sédiments (Golterman \& de Oude 1991). Généralement, on s'intéresse peu à la chimie de ce processus ; les études actuelles concernent principalement les échanges eau-sédiment et l'approche est de type : « boite noire ». Néanmoins, nous pensons que le devenir du phosphate dans les lacs ne peut être compris qu'à travers une connaissance des processus chimiques réglant les mécanismes d'adsorption et de précipitation. Ce ne sera qu'à partir de résultats précis obtenus sur un lac que l'on pourra extrapoler à d'autres lacs.

Cet article est une synthèse de nos travaux effectués en Camargue et dont l'objectif est d'arriver à une quantification des flux de phosphate. Notre travail porte d'abord sur l'adsorption du phosphate sur les hydroxydes ferriques $(\mathrm{Fe}(\mathrm{OOH})$ ) ainsi que sur la précipitation du phosphate de calcium $\left(\mathrm{CaCO}_{3} \approx \mathrm{P}\right)$. Au cours de cette étude, de nouveaux aspects du diagramme de solubilité des deux systèmes sont proposés. De plus, l'identification des phosphates organiques a également nettement progressé.

Le schéma utilisé pour la détermination du phosphate lié au fer $(\mathrm{Fe}(\mathrm{OOH}) \approx \mathrm{P})$ et du phosphate lié au calcium $\left(\mathrm{CaCO}_{3} \approx \mathrm{P}\right)$ doit être décrit pour aborder la discussion. Le principe est d'utiliser des agents chélateurs pour réaliser les extractions successives. (De Groot \& Golterman 1989, 1990). Le $\mathrm{Fe}(\mathrm{OOH}) \approx \mathrm{P}$ est extrait avec une solution de $\mathrm{Ca}$ NTA et de dithionite puis le $\mathrm{CaCO}_{3} \approx \mathrm{P}$ avec une solution de Na-EDTA. Chaque extractant est appliqué plusieurs fois sur le même culot jusqu'à épuisement du phosphate lié au fer (Ca-NTA) et du phosphate lié au calcium (Na-EDTA). Dans les deux extraits on trouve généralement très peu de phosphate relié à la matière organique (moins de $3 \%$ du $\mathbf{P}_{\text {tot }}$ ). Les analyses sont reproductibles et les extractions réalisées sur des composés bien définis et préparés en laboratoire, donnent les résultats attendus.

Après extraction du $\mathbf{P}$ inorganique, une quantité importante de $P$ resté fixée dans le sédiment. Les agents chélateurs étant moins agressifs que les produits utilisés classiquement pour les extractions ( $\mathrm{NaOH}$ et $\mathrm{H}_{2} \mathrm{SO}_{4}$ ), nous pensons que le pool restant est organique, et quantitativement plus important qu'il ne l'a été souvent supposé (de Groot \& Golterman 1990). Une grande partie de ce pool restant peut être extraite à l'acide (P-org acidosoluble, POAS) ; cette fraction a été supposée être du $\mathrm{CaCO}_{3} \approx \mathrm{P}$ dans les schémas classiques d'extraction. Après l'extraction du POAS, il reste toujours du phosphate dans le culot, qui peut être presque entièrement extrait avec le $\mathrm{NaOH}$. La composition de cette dernière fraction est élucidée. Le schéma complet est le suivant :

\section{Sédiment \\ $\downarrow$ extraction avec Ca-NTA $\rightarrow \mathrm{Fe}(\mathrm{OOH}) \approx \mathrm{P}$ \\ Culot I \\ $\downarrow$ extraction avec EDTA $\rightarrow \mathrm{CaCO}_{3} \approx \mathrm{P}$ \\ Culot II \\ $\downarrow$ extraction avec $0,5 \mathrm{M} \mathrm{HCl} \rightarrow$ POAS (P humique ? $30 \mathrm{~min} \quad$ voir ci-dessous) \\ Culot IV (P organique restant) \\ $\downarrow$ extraction avec $2 \mathrm{M} \mathrm{NaOH} \rightarrow$ POR (P org restant ; $\left(90^{\circ} \mathrm{C}, 5 \mathrm{~min}\right) \quad$ voir ci-dessous)}

Culot V ( \pm sans phosphate)

\section{L'adsorption des phosphates}

Le phosphate entrant dans un système aquatique est distribué dans les deux compartiments, eau et sédiment, de la façon suivante :

$$
P_{\text {in }}=P_{\text {sed }}+P_{\text {out }}+\Delta(o-P)
$$

où

$P_{\text {in }}=$ phosphate entrant dans le lac $\left(\mathrm{g} \mathrm{m}^{-2} \mathrm{a}^{-1}\right)$

$\mathbf{P}_{\text {out }}=$ phosphate sortant du lac $\left(\mathrm{g} \mathrm{m}^{-2} \mathrm{a}^{-1}\right)$

$P_{\text {sed }}=$ phosphate retenu dans les sédiments $\left(\mathrm{g} \mathrm{m}^{-2} \mathrm{a}^{-1}\right)$

$\Delta(\mathrm{o}-\mathrm{P})=$ augmentation de la concentration en phosphate dans l'eau * profondeur $\left(\mathrm{g} \mathrm{m}^{-2} \mathrm{a}^{-1}\right)$

Le phosphate entrant dans le sédiment ne provient pas seulement de la dégradation de la matière organique, il y a également adsorption chimique. Dans un résumé de ce processus, Golterman (1984) suggère de décrire l'équilibre d'adsorption par l'isotherme d'adsorption de Freundlich, ce qui avait été proposé par Olsen (1964) :

$$
P_{\text {sed }}=A^{*}(0-P)^{B}
$$

où

$P_{\text {sed }}=$ concentration de phosphates dans le sédiment (mg.g $\left.{ }^{-1}\right)$

(o-P) $=$ concentration de phosphates dans l'eau (mg. 1-1) $^{-1}$ 
A et $\mathbf{B}$ sont des constantes :

- B est généralement comprise entre 0,3 et 0,4

- A est reliée à la capacité d'adsorption des sédiments et est reliée aux concentrations en $\mathrm{Ca}^{2+}$, en $\mathrm{Fe}(\mathrm{OOH})$ et au $\mathrm{pH}$.

Deux exemples d'isothermes d'adsorption de phosphate sont donnés sur la Fíg. 1.

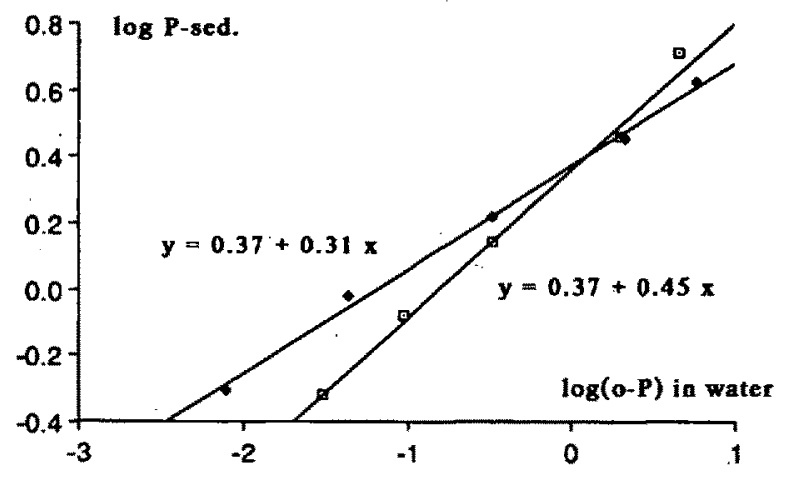

Fig. 1. Adsorption de l'ortho-phosphate sur les sédiments du Lac Puyvalador (Pyrénées) et d'une rizière dans le bassin versant du Pô. Transformation $\log / \mathrm{log}$ des données de Golterman, 1984. Concentration de l'o-P et de $P_{\text {sed }}$ en $\mathrm{mg} \mathrm{1}^{-1}$ (les deux $\mathrm{R}^{2}>0.99$ ).

Fig. 1. Ortho-phosphate adsorption onto sediments from Lake Puyvalador (Pyrenees) and from a ricefield in the river Po watershed. Log transformation of data from Golterman, 1984. (0-P and $P_{\text {sed }}$ in $\mathrm{mg}^{-1}$ ) (Both $R^{2}>0.99$ ).

Kuo \& Lotse (1974), étudiant la cinétique de l'adsorption du phosphate sur deux sols, ont également montré que l'isotherme de Freundlich décrit bien cette adsorption. Cependant, les concentrations utilisées par ces auteurs sont si élevées que leurs résultats ne peuvent pas être directement comparés aux nôtres (voir Fig. 2).

L'importance de $\mathrm{Fe}(\mathrm{OOH})$ comme adsorbant dans les sédiments est connue depuis longtemps (Mortimer 1941, 1942, Olsen 1964, Lijklema 1977). Fox (1993) a démontré la présence d'un complexe de $\mathrm{Fe}(\mathrm{OOH})$ et o-P dans plusieurs fleuves avec des concentrations basses en $\mathrm{Ca}^{2}+$. La structure de ce complexe est cependant encore mal connue. Syers \& Curtin (1989), à la suite des études d'absorption infrarouge de Parfitt et al (1976) ont proposé un complexe binucléaire de structure suivante :

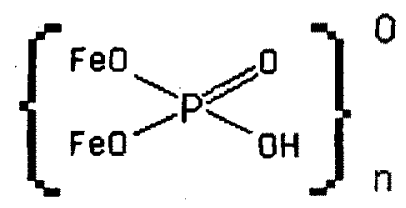

mais il faut signaler que les spectres infrarouge sont toujours faits sur des échantillons séchés, ce qui change leur structure.

L'équilibre entre le $\mathrm{Fe}(\mathrm{OOH})$ et l'o-P peut être décrit par l'équation empirique de Freundlich (II). Lijklema (1977) a été le premier à faire une approche quantitative en utilisant une description logarithmique qui tenait compte de l'influence du $\mathrm{pH}$ :

$$
\mathrm{P}_{\mathrm{ads}} / \mathrm{Fe}=0,298-0,031^{*} \mathrm{pH}+0,201^{*}(\mathrm{P})^{0,5} \text {. }
$$

Ses données ont été recalculées par Golterman (1982) en utilisant l'équation empirique de Freundlich (eq. II) :

$$
\begin{aligned}
& \mathrm{P}_{\text {ads }} / \mathrm{Fe}=\mathrm{A}^{*}(\mathrm{o}-\mathrm{P})^{0.2} \\
& \text { avec } \mathrm{A}=\left(\mathrm{a}+\mathrm{b}^{*} \mathrm{pH}\right)
\end{aligned}
$$

Puisque l'estimation de la constante A de Freundlich est nécessaire pour le calcul de l'équilibre entre l'o-P contenu dans l'eau et celui contenu dans les sédiments, ceci en fonction du $\mathrm{pH}$ et de la concentration en $\mathrm{Ca}^{2}+$ dissous, les valeurs des constantes $A$ et $\mathrm{B}$ ont été de nouveau déterminées pour différentes valeurs de $\mathrm{pH}$ par ajustement de courbes expérimentales (Golterman 1994a). Quelques exemples sont représentés sur la Figure 2. Tous les résultats calculés sont donnés dans le Tableau 1 .

Avec les données du Tableau 1, on peut d'abord ajuster la relation entre $\mathrm{A}$ et le $\mathrm{pH}$ sans tenir compte de $\mathrm{Ca}^{2+}$. Deux ajustements utilisant soit une loi exponentielle, soit un polynome d'ordre 2

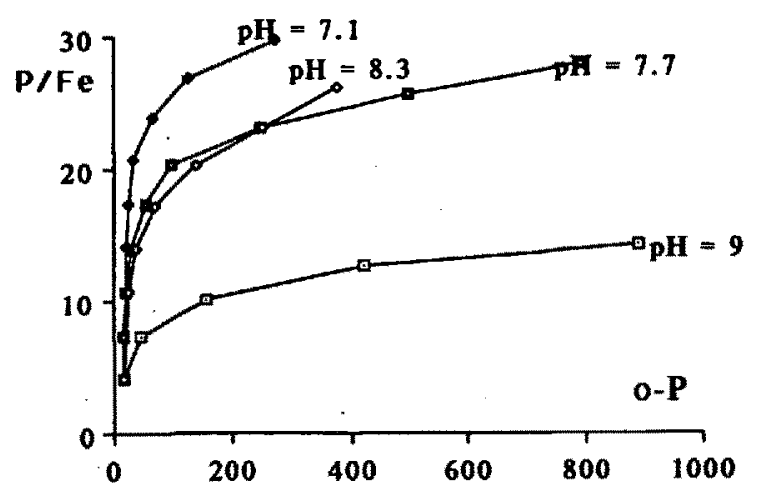

Fig. 2. Adsorption de l'ortho-phosphate sur le $\mathrm{Fe}(\mathrm{OOH})$. Axe $\mathrm{Y}: \mu \mathrm{g} \mathrm{P}$ par $\mathrm{mg} \mathrm{Fe}$; Axe X : concentration de l'ortho-

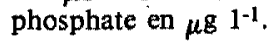

Fig. 2. Adsorption of ortho-phosphate onto $\mathrm{Fe}(\mathrm{OOH})$. $\mathrm{Y}$ axis : $\mu \mathrm{g} P$ per mg Fe ; $\mathrm{X}$ axis : ortho-phosphate concentration in

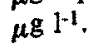


conduisent à de bonnes estimations de la valeur $\mathrm{A}$ $\left(\mathrm{R}^{2}>0.9\right)$. Nous avons retenu la courbe exponentielle :

$$
\mathrm{A}=\mathrm{a} \cdot 10^{-0.42 * \mathrm{pH}} \text {. ou } \mathrm{A}=\mathrm{a} \cdot\left[\mathrm{H}^{+}\right]^{0.42}
$$

(La relation avec $\left[\mathrm{H}^{+}\right]$peut être attribuée à ce que entre $\mathrm{pH}=5$ et 9 , le rapport $\mathrm{H}_{2} \mathrm{PO}_{4}{ }^{-} / \mathrm{HPO}_{4}{ }^{2-}$ dépend linéairement de $\left[\mathrm{H}^{+}\right]$. Quant à la constante 0.42 , on ne peut pas encore lui attribuer de signification chimique).

La constante B varie peu, mais il n'y a aucune relation avec le $\mathrm{pH}$. On perd très peu de précision si $B$ est ajusté à $\mathbf{0 . 3 3 3}$ avec le polynôme, mais on gagne avec la courbe exponentielle (voir Tableau 1).

Dans un second temps nous avons inclus la concentration du $\mathrm{Ca}^{2}+$, dans une simple régression multiple. L'équation $\mathrm{A}=\mathrm{C}_{1}+\mathrm{C}_{2}{ }^{*} \mathrm{pH}+\mathrm{C}_{3}{ }^{*} \mathrm{e}^{-\mathrm{Ca}}$ donne un résultat numérique satisfaisant, pour chaque expérience sauf pour les valeurs extrêmes $(\mathrm{N}$ $=24 ; R^{2}=0.9$ ). Ensuite, nous avons ajusté toutes les données avec

$$
A=\text { a.10b.pH }\left\{C_{1}-\left(C_{1}-1\right) \cdot e^{-C a}\right\}
$$

Cette équation redonne la courbe exponentielle (IV) trouvée pour $\mathrm{Ca}=0$, ce qui implique l'hypothèse que l'influence de $\mathrm{Ca}^{2}+$ suit une hyperbole, avec $C_{1}$ comme asymptote pour les concentrations de $\mathrm{Ca}^{2}+$ élevées. (Le désavantage de cette courbe est que l'ajustement doit être fait après transformation $\log / \log$ ). Nous avons trouvé un $\mathrm{R}^{2}=0.74$ pour $\mathrm{N}=32$ et un $\mathrm{R}^{2}=0.9$, si les données de l'été sont exclues (Golterman 1994a). Utilisant toutes les données on trouve l'équation :

$\log \mathrm{A}=2.0462-0.13174^{*} \mathrm{pH}+0.1549^{*}\left(2.89-1.89 . \mathrm{e}^{-\mathrm{Ca}}\right)$

La constante A peut être utilisée maintenant pour reconstruire le diagramme de solubilité de l'o-P.

Tableau 1. Constantes de Freundlich pour les équilibres d'adsorption de l'o-P sur $\mathrm{Fe}(\mathrm{OOH})\left(300 \mathrm{mg} \mathrm{l}^{-1}\right.$ de $\left.\mathrm{Fe}^{3+}\right)$ à diffèrentes valeurs de pH. Courbes avec ajustement majeur et courbes $\log / \log$ forcées par $(0,0)$ et $\mathrm{B}=0.333$. $\mathrm{Ca}^{2+}=1 \mathrm{mMol}^{-1}$

Table 1. Freundlich's constants for adsorption equilibria of o-P onto $\mathrm{Fe}(\mathrm{OOH})\left(300 \mathrm{mg}^{-1} \mathrm{of} \mathrm{Fe}^{3+}\right)$ at different pH values. Results

\begin{tabular}{|c|c|c|c|c|c|c|}
\hline \multicolumn{4}{|c|}{$\begin{array}{l}\text { meilleur ajustement } \\
\text { best fit }\end{array}$} & \multicolumn{3}{|c|}{$\begin{array}{l}\text { forcée par }(0,0) \text { et } B=0.333 \\
\text { forced through }(0,0) \text { and } B=0.333\end{array}$} \\
\hline $\begin{array}{l}\mathrm{pH} \\
7.04 \\
7.1 \\
7.7 \\
8.0 \\
8.3 \\
9.0 \\
9.0 \\
9.6 \\
11.0 \\
11.0\end{array}$ & $\begin{array}{c}\text { A } \\
15.9 \\
14.4 \\
10.1 \\
9.3 \\
11.1 \\
4.21 \\
4.22 \\
4.12 \\
0.26 \\
0.36\end{array}$ & $\begin{array}{l}B \\
0.28 \\
0.28 \\
0.25 \\
0.27 \\
0.30 \\
0.21 \\
0.27 \\
0.22 \\
0.57 \\
0.70\end{array}$ & \begin{tabular}{l}
\multicolumn{1}{c}{$\mathrm{R}^{2}$} \\
0.92 \\
0.92 \\
0.93 \\
0.99 \\
0.98 \\
0.999 \\
0.99 \\
0.99 \\
0.97 \\
0.99
\end{tabular} & $\begin{array}{c}\text { A } \\
16.8 \\
16 \\
10.5 \\
9.73 \\
11.5 \\
3.92 \\
4.1 \\
3.97 \\
0.39 \\
0.65\end{array}$ & $\begin{array}{c}\mathbf{R}^{2} \\
0.84 \\
0.85 \\
0.84 \\
0.99 \\
0.96 \\
0.84 \\
0.96 \\
0.92 \\
0.82 \\
0.90\end{array}$ & \\
\hline \multicolumn{3}{|c|}{$\begin{array}{l}\mathrm{A}=91.1-15.5^{*} \mathrm{pH}+0.65^{*} \mathrm{pH}^{2} \\
\mathrm{~A}=22192^{*} 10^{(-0.42 \mathrm{pH})}\end{array}$} & $\begin{array}{l}\mathbf{R}^{2}=0.95 \\
\mathbf{R}^{2}=0.91\end{array}$ & $\begin{array}{l}A=192.5-35 \\
A=23626 * 10\end{array}$ & $\begin{array}{l}+1.65 \mathrm{pH}^{2} \\
* \mathrm{pH})\end{array}$ & $\begin{array}{l}\mathrm{R}^{2}=0.87 \\
\mathrm{R}^{2}=0.96\end{array}$ \\
\hline
\end{tabular}
for best fitted curves and for curves after $\log / \log$ transformation forced through $(0.0)$ and $B=0.333$. $\mathrm{Ca}^{2+}=1 \mathrm{mMol}^{-1}$.

Toutes les données des expériences avec $\mathrm{Ca}^{2+}\left(0-2 \mathrm{mmol} \mathrm{l}^{-1}\right)$ :

$\log \mathrm{A}=2.05643-0.131737^{*} \mathrm{pH}+0.289246 *\left(2-\mathrm{e}^{-\mathrm{Ca}}\right) \quad \mathrm{R}^{2}=0.74$ 
Nos résultats sont proches de ceux de Barrow (1985) qui, en utilisant un modèle plus compliqué, a montré également que l'adsorption dépend du $\mathrm{pH}$, mais pas du $\mathrm{Ca}^{2}+$. Les constantes de l'isotherme de Freundlich ne sont pas fournies dans leurs résultats. D'autres auteurs (Stumm et al. 1980 : Goldberg \& Sposito 1984) ont proposé un modèle assez compliqué, comme le « Constant Capacitance Model ». Ce modèle est très complexe et n'a pas encore donné de résultats applicables comme le modèle simple de Freundlich.

Sverjensky (1993) a critiqué le " CCM » et a proposé un modèle basé sur la théorie de Born de la solvatation ionique utilisant l'énergie libre de Gibbs. Ce modèle n'a été utilisé que pour les cations ; il explique l'adsorption de $\mathrm{Ca}^{2}+$ sur le $\mathrm{Fe}(\mathrm{OOH})$, ce qui met en évidence l'influence de $\mathrm{Ca}^{2}+$ sur l'adsorption de o-P par le $\mathrm{Fe}(\mathrm{OOH})$.

La question est de savoir pourquoi le modèle empirique de Freundlich s'accorde si bien aux données observées, alors qu'il n'est basé sur aucune loi chimique ou physique. Par une étude théorique, nous avons pu montrer que l'équation (II) est mathématiquement une bonne approche si nous supposons que pour une molécule de $\mathbf{P}$ adsorbée, deux molécules de $\mathrm{Fe}(\mathrm{OOH})$ sont rendues inactives. Cette supposition s'accorde bien avec le « inner sphere surface complex " de l'adsorption de phosphate sur la goethite comme proposé par Sposito (1989). Une formule mathématique complexe est obtenue : dans certaines conditions, elle peut être simplifiée à l'équation (II). Ceci est un argument essentiel contre le modèle de Langmuir qui suppose un nombre maximal de site d'adsorption. Dans les sédiments, la quantité de $\mathrm{Fe}(\mathrm{OOH})$ libre règle la capacité d'adsorption, c'est-à-dire « $a$ » et « $b$ », et par conséquent « $\mathrm{A}$ ». La quantité de $\mathrm{Fe}(\mathrm{OOH}) \approx \mathrm{P}$ dans les sédiments peut être estimée par extraction au Ca-NTA dans des conditions réductrices (Golterman \& Booman 1988, De Groot \& Golterman 1990). Nous ne savons pas dans quelle mesure les complexes humiques $\mathrm{Fe}-\mathrm{P}$ sont impliqués; les extraits obtenus avec le Ca-NTA sont toujours de couleur brun foncé et des interactions paraissent possibles.

Dans les lacs peu profonds et les marais, le $\mathrm{Fe}(\mathrm{OOH})$ ne représente qu'un faible pourcentage du fer total (5-10\%); la majeure partie est incluse dans l'argile ou se trouve sous forme de FeS. Des transitions d'une forme à une autre, dépendant des changements du potentiel rédox et du $\mathrm{pH}$, ont probablement lieu. C'est pour cette raison que nous avons converti le $\mathrm{Fe}(\mathrm{OOH})$ des suspensions de sédiments (provenant de marais en Camargue et du lit de la Garonne) en FeS, par addition de différentes quantités de $\mathrm{Na}_{2} \mathrm{~S}$. Les changements de concentration en o-P et en $\mathrm{Fe}(\mathrm{OOH}) \approx \mathrm{P}$ ont été suivis dans le temps. On observe une augmentation de la teneur en o-P dans l'eau plus faible que prévue, ainsi que la présence constante d'une fraction de $\mathrm{Fe}(\mathrm{OOH}) \approx \mathrm{P}$ même quand $\mathrm{Na}_{2} \mathrm{~S}$ est ajouté en excès. Ceci est supposé être causé par la formation d'un sel de fer et de phosphate $\left(\mathrm{FePO}_{4}\right.$ ou $\mathrm{Fe}_{3}\left(\mathrm{PO}_{4}\right)_{2}$ vivianite) pendant la réduction de $\mathrm{Fe}(\mathrm{OOH})$ (De Groot 1991).

D'autres expériences ont été réalisées : suivi de la teneur en o-P lors de la réduction d'une suspension de $\mathrm{Fe}(\mathrm{OOH}) \approx \mathrm{P}$ : suivi de la teneur en o-P lors de l'oxydation d'une suspension de FeS ; elles confirment les résultats des précédentes expériences (De Groot 1991).

Nous pensons qu'il est probable que les changements dans le système $\mathrm{FeS} / \mathrm{Fe}(\mathrm{OOH})$ ont une influence significative sur l'adsorption des o-P plus marquée dans les lacs peu profonds et les marais que dans les lacs profonds.

\section{Le processus de précipitation}

Le deuxième facteur, réglant l'équilibre des o-P entre l'eau et les sédiments, est la concentration en $\mathrm{Ca}^{2}+$ de l'eau. On sait que la faible solubilité de l'apatite, $\mathrm{Ca}_{5}\left(\mathrm{PO}_{4}\right)_{3} \mathrm{OH}$, régit la concentration d'o-P dans l'eau (Golterman 1984). La valeur précise du produit de solubilité est longtemps restée un problème. Des valeurs de $10^{-50}$ à $10^{-60}$ ont été proposées. Une solution pratique a été donnée par Golterman \& Meyer (1985) qui ont déterminé le produit ionique $\mathrm{PI}=(\mathrm{Ca})^{5}\left(\mathrm{PO}_{4}\right)^{3}(\mathrm{OH})$ à partir de 924 échantillons provenant de deux fleuves à eau calcaire, le Rhin et le Rhône. Ils ont trouvé une valeur moyenne de $10^{-50}$ avec un écart-type de $\left(10^{-0}, 3\right)$. D'autres auteurs ont confirmé que cette valeur peut être utilisée pour calculer la solubilité du phosphate en fonction du $\mathrm{pH}$ et de la concentration de $\mathrm{Ca}^{2}+$ (Staudinger et al. 1990, López \& Morgui 1992, Moutin 1992). Nous pensons qu'il est raisonnable d'utiliser ce produit de solubilité pour déterminer la concentration en o-P d'une eau calcaire en 
équilibre avec l'air. La précision de ce calcul a été discutée par Clymo \& Golterman (1985). Deux incertitudes restent encore à élucider : l'influence des coefficients d'activité ionique et l'incertitude sur la valeur du $\mathrm{K}_{3}$ de l'acide phosphorique. A la suite d'une étude bibliographique, Ghosh et al. (1980) ont montré que cette valeur variait entre 2 et $20 \times 10^{-13}$. Il est étonnant qu'aussi peu de mesures précises de cette constante aient été réalisées.

Arvieu (1981), Syers \& Curtin (1989) ont également montré que l'apatite est le composé le moins soluble en milieu calcaire, alors que dans les sols acides la solubilité du phosphate peut être également contrôlée par d'autres phases solides (Lindsay 1989). La différence entre les sédiments (sous l'eau) et les sols (et entre les différents sols eux-mêmes) peut être expliquée par le fait que les sédiments sont plus proches d'un équilibre thermodynamique vis-à-vis de l'adsorption de l'o-P que les sols.

\section{Vue d'ensemble sur l'adsorption et la précipitation}

Golterman (1988a) a calculé simultanément dans un diagramme de solubilité, les équilibres avec le $\mathrm{Fe}(\mathrm{OOH}) \approx \mathrm{P}$ d'une part et avec le $\mathrm{CaCO}_{3} \approx \mathrm{P}$ d'autre part. Ce diagramme décrit la solubilité de l'o-P en fonction du $\mathrm{pH}$, de la concentration en $\mathrm{Fe}(\mathrm{OOH})$ dans le sédiment et de la concentration en calcium dans l'eau. La concentration en $\mathrm{CaCO}_{3}$ dans les sédiments n'intervient pas (Fig. 3).

Ce diagramme devait servir de première approche pour modéliser l'équilibre entre les sédiments et l'eau surnageante dans le cas des eaux calcaires. Des développements récents ont toutefois montré qu'il devait être corrigé. Nos expériences sur l'adsorption ont toujours été réalisés avec du $\mathrm{Fe}(\mathrm{OOH})$ dans de $l^{\prime} \mathrm{H}_{2} \mathrm{O}$; nous avons maintenant montré que le $\mathrm{Na}^{+}$, le $\mathrm{Mg}^{2}+$ et le $\mathrm{Ca}^{2}+$ (sous formes de chlorures) peuvent augmenter considérablement l'adsorption des o-P sur le $\mathrm{Fe}(\mathrm{OOH})$. A des concentrations ordinaires, la quantité d'o-P restant en solution peut être diminuée de $50 \%$. Pour le $\mathrm{NaCl}$ et le $\mathrm{MgCl}_{2}$, cette augmentation peut être quantifiée. Par contre, il n'est pas possible de distinguer l'influence du $\mathrm{Ca}^{2}+$ sur le processus d'adsorption de la précipitation de $\mathrm{CaCO}_{3} ;$ le Ca${ }^{2}+$ ajouté au mélange disparaît de la solution. Un travail dans cette direction est en cours, mais il est difficile car pour des concentrations en

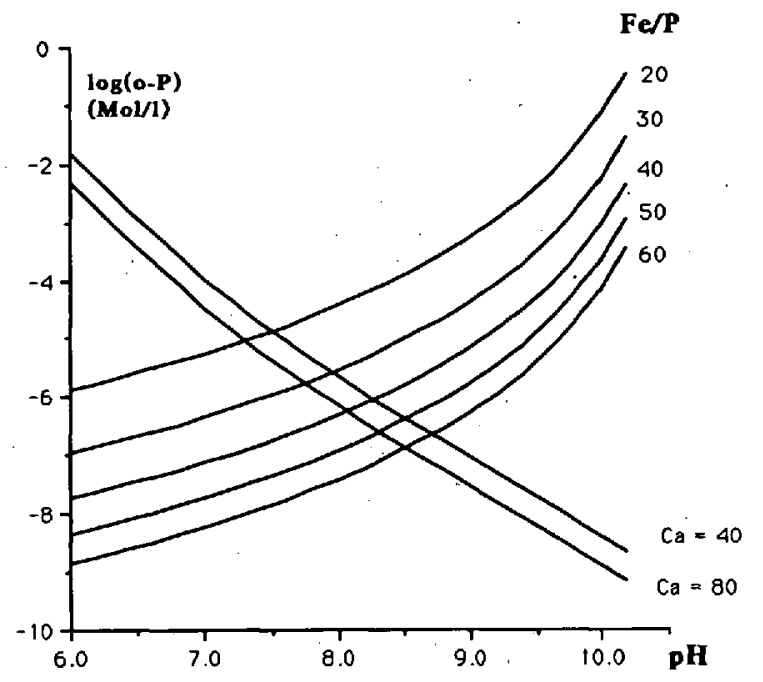

Fig. 3. Diagramme de solubilité de l'o-P en fonction du $\mathrm{pH}$, concentration de $\mathrm{Ca}^{2}+$ dans l'eau et rapport $\mathrm{Fe}(\mathrm{OOH}) / \mathrm{P}$ dans les sédiments (Golterman 1988).

Fig. 3. Solubility diagramme of o-P as function of $\mathrm{pH}$, concentration of $\mathrm{Ca}^{2+}$ in the water and $\mathrm{Fe}(\mathrm{OOH}) / \mathrm{P}$ ratio in the sediments (Golterman 1988).

o-P et des valeurs de $\mathrm{pH}$ faibles, les o-P sontadsorbés si fortement qu'il n'en reste que très peu en solution alors que pour des concentrations en O-P et des valeurs de $\mathrm{pH}$ élevées, la précipitation d'apatite devient importante.

\section{Les phosphates organiques}

Dans le passé, les essais d'extractions des phosphates organiques ont souvent échoué car l'o-P formé lors de l'hydrolyse était rapidement réadsorbé sur le $\mathrm{Fe}(\mathrm{OOH})$. Le $\mathrm{Fe}(\mathrm{OOH})$ et le $\mathrm{CaCO}_{3}$ étant préalablement extraits dans notre schéma d'extraction (par le NTA et l'EDTA), cette source d'erreur n'existe plus. Puiqu'avec le NTA et l'EDTA, il n'est extrait que de faibles quantités de P-org et qu'il est peu probable que des composés inorganiques restent dans le culot, on suppose généralement que le $P$ restant est organique. De Groot (1990) et De Groot \& Golterman (1993) ont pu approfondir l'analyse de deux pools des phosphates organiques.

La première extraction du P-org est réalisée avec un acide fort (par exemple $\mathrm{HCl} 0.5 \mathrm{M}$ ) pendant une période relativement courte $(30 \mathrm{mn})$. Une partie importante du P-org est ainsi extraite (POAS). 
La concentration de ce pool variait entre $P=60$ et $225 \mu \mathrm{g} . \mathrm{g}^{-1}$. Etant donné que les phosphates sont libérés sous forme d'o-P, nous n'avons pas de preuve que ce $\mathrm{P}$ soit lié à des composés humiques, bien que ceci paraisse très probable. Il est intéressant de noter que l'acide n'extrait que très peu de ce type de matière, tandis que $\mathrm{H}_{2} \mathrm{O}$ peut en extraire beaucoup plus. Cela signifie que l'acide modifie la solubilité des matières humiques. En effet, après une extraction avec l'acide, $\mathrm{NaOH}$ extrait encore plus de matière brune, qu'avant une extraction avec l'acide. Bien que Gjessing (1976) et Frimmel \& Christman (1988) dans leurs traités sur la Matière Humique (MH) discutent ce complexe, celui-ci n'a pas été encore mis en évidence par expérimentation ; Gjessing (1976), en utilisant la filtration sur gel, a isolé une fraction dissoute de haut poids moléculaire et qui contenait du phosphate. Batsula \& Krivonosova (1973) ont isolé un acide fulvique contenant de 2 à $4 \%$ de $P$ et pensent que ce $P$ se trouvait dans la chaîne latérale de cet acide. Francko \& Heath (1983) ont montré par double chromatographie (Dowex AG1-X8 et Sephadex G-25) l'existence d'un complexe de $\mathrm{MH} / \mathrm{Fe}^{3}+/ \mathrm{PO}_{4}$ dissous dans l'eau d'un marais acide $(\mathrm{pH}=5)$. Ils ont montré que le phosphate et la $\mathrm{MH}$ sont reliés au $\mathrm{Fer}^{3+}$, mais que ce complexe est photolabile.

Tipping \& Woof (1983) et Tipping (1985) ont montré une co-accumulation entre matière humique $(\mathrm{MH})$ et $\mathrm{Fe}(\mathrm{OOH})$ et une corrélation entre $\mathrm{MH}$ et $\mathrm{Fe}(\mathrm{OOH})$ dans l'hypolimnion de Estwaite Water (Angleterre). On peut supposer que ce même complexe peut également adsorber de l'o-P, donnant un complexe $\mathrm{MH} / \mathrm{Fe}(\mathrm{OOH}) / \mathrm{PO}_{4}$, dont la partie minérale serait soluble par action d'un acide fort.

Tipping \& Cooke (1982) ont montré que des particules de $\mathrm{Fe}(\mathrm{OOH})$ obtiennent une charge négative par l'adsorption de MH. L'importance de cette charge diminuait avec des concentrations élevées de $\mathrm{Ca}^{2}+$ et $\mathrm{Mg}^{2}+$. Ils discutent les interactions possibles avec l'o-P, mais n'ont pas montré l'existence du complexe : $\mathrm{MH} / \mathrm{Fe}(\mathrm{OOH}) / \mathrm{PO}_{4}$.

Buffle (1988), dans son traité sur les réactions de complexation, ne traite que des réactions d'équilibre entre $\mathrm{Fe}(\mathrm{OOH})$ et le phosphate, mais il ne discute pas la structure de ce complexe. Un complexe organique n'est même pas du tout mentionné.

Cè pool de phosphate (le POAS) a été rapidement reminéralisé pendant l'assèchement d'un marais en
Camargue (De Groot \& Fabre, 1993). Bien que le POAS ne soit pas directement biodisponible, l'o-P produit est utilisable pour la végétation ; l'assèchement fait augmenter la quantité du phosphate disponible.

Le $P$ restant dans le culot peut ensuite être extrait avec une solution de $\mathrm{NaOH}\left(2 \mathrm{M}\right.$ à $\left.90^{\circ} \mathrm{C}\right)$. La concentration de ce pool variait entre $P=100$ et $300 \mu \mathrm{g} \cdot \mathrm{g}^{-1}$. Dans ce cas, le $\mathrm{P}$ passant en solution est sous forme de P-org et nous avons observé qu'il pouvait être hydrolysé en o-P par la « phytase 2 ». Nous avons également observé que la phosphatase alcaline ne libérait pas d'o-P du culot. Ceci nous permet de suggérer que ce $\mathrm{P}$-org est de la phytine ('exaphosphate d'inositol). La concentration minimale variait entre $P=25$ et $150 \mu \mathrm{g} \cdot \mathrm{g}^{-1}$.

Des expériences avec des composés préparés au laboratoire, ont montré qu'il s'agissait probablement du $\mathrm{Fe}_{4}$-phytate. Ce composé a été préparé par adsorption de la phytine sur le $\mathrm{Fe}(\mathrm{OOH})$. Il est également insoluble dans l' $\mathrm{HCl}(0,5 \mathrm{M})$, alors que les sels de phytate avec $\mathrm{Ca}^{2}+$ et $\mathrm{Mg}^{2}+$ sont solubles dans l'acide. D'autre part, le $\mathrm{Fe}_{4}$-phytate préparé au laboratoire n'est pas extrait par le NTA, ni par l'EDTA, ni par l'HCL $0.5 \mathrm{M}$, mais uniquement par le $\mathrm{NaOH} 2 \mathrm{M}$ à $90^{\circ} \mathrm{C}$. Le $\mathrm{Fe}(\mathrm{OOH})$ lié à la phytine paraît protégé contre le pouvoir acide ou chélateur des extractants alors que le $\mathrm{Fe}(\mathrm{OOH})$ libre passe en solution lors d'une attaque acide. L'analyse du précipité blanc ainsi formé a un rapport $\mathrm{Fe} / \mathrm{P}$ de 4,3/6 ( $\mathrm{mol} / \mathrm{mol})$. Les quantités du phytate trouvées dans nos sédiments sont comparables aux quantités trouvées dans des sols. (Pour comparaison, voir De Groot \& Golterman 1993).

Dans le passé, on s'est demandé pourquoi les sédiments pouvaient contenir autant de $\mathrm{P}$-org, la plupart des composés organiques étant biodégradables. La présence de phosphates liés aux substances humiques et de phosphate d'inositol adsorbé sur le $\mathrm{Fe}(\mathrm{OOH})$ explique ce fait, car ces deux composés sont très résistants aux attaques bactériennes.

Nous pensons avoir obtenu des preuves suffisantes, bien que non encore définies, de la présence de phosphates liés aux substances humiques et de $\mathrm{Fe}_{4}$-phytate dans la fraction P-org des sédiments. La poursuite des travaux sur l'identification des molécules composant la fraction organique, ainsi que l'analyse d'autres sédiments, paraît primordiale. 


\section{Etudes sur la biodisponibilité}

La connaissance des quantités de $\mathrm{Fe}(\mathrm{OOH}) \approx \mathrm{P}$ et de $\mathrm{CaCO}_{3} \approx \mathrm{P}$ est nécessaire pour estimer la disponibilité des phosphates des sédiments pour la croissance du phytoplancton. Il est souvent suggéré que seul le $\mathrm{Fe}(\mathrm{OOH}) \approx \mathrm{P}$ est disponible, tandis que le $\mathrm{CaCO}_{3} \approx \mathrm{P}$ ne l'est pas. De Graaf BierbrauwerWürtz \& Golterman (1989) ont toutefois montré qu'il existait une forte corrélation entre la somme $\left(\mathrm{Fe}(\mathrm{OOH}) \approx \mathrm{P}+\mathrm{CaCO}_{3} \approx \mathrm{P}\right)$ et le $\mathrm{P}$ disponible pour certaines espèces de Scenedesmus en culture (Voir Fig. 4). Des travaux récents ont montré que le $P$ restant après extraction des $(\mathrm{Fe}(\mathrm{OOH}) \approx \mathrm{P}+$ $\mathrm{CaCO}_{3} \approx \mathrm{P}$ ) n'est pas disponible pour ces espèces de Scenedesmus. Le POAS peut devenir biodisponible après reminéralisation, par exemple après l'assèchement des sédiments.

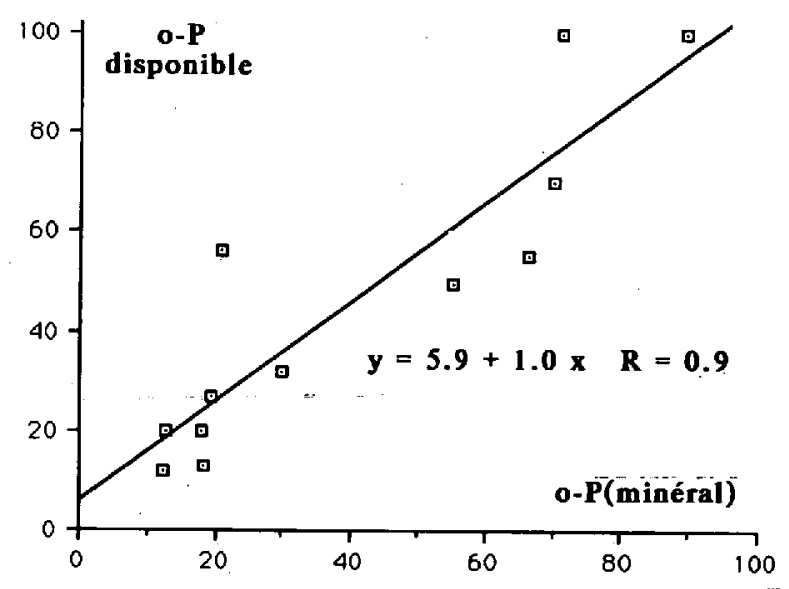

Fig. 4. Corrélation entre o-P biodisponible et $(\mathrm{Fe}(\mathrm{OOH}) \approx \mathbf{P}+$ $\mathrm{CaCO}_{3} \approx \mathrm{P}$ ) dans 11 sédiments hollañdais (o-P en $\mu \mathrm{g} \mathrm{l}^{-1}$ ).

Fig. 4. Correlation between available o-P and $(\mathrm{Fe}(\mathrm{OOH}) \approx \mathbf{P}+$ $\mathrm{CaCO}_{3} \approx \mathrm{P}$ ) in 11 dutch lakes (o-P in $\mu \mathrm{g} \mathrm{t}^{-1}$ ).

Dans certains fleuves et réservoirs en Afrique du Sud Grobler \& Davie $(1979,1981)$ ont également montré que le phosphate extractible avec le NTA (donc $\mathrm{Fe}(\mathrm{OOH}) \approx \mathrm{P}$ et $\mathrm{CaCO}_{3} \approx \mathrm{P}$ éventuellement présents) était correlé $(R=0.97)$ avec le phosphate disponible pour Selenastrum capricornutum en culture. Grobbelaar (1983) a montré que dans l'Amazone, dont l'eau contient peu de $\mathrm{Ca}^{2+}$, la plupart du phosphate particulaire (donc adsorbé sur les sédiments comme $\mathrm{Fe}(\mathrm{OOH}) \approx \mathrm{P}$ ) était disponible pour le phytoplancton.
Pour une discussion plus détaillée sur la disponibilité du P des sédiments, voir Golterman (1988 b).

\section{Accumulation en lacs peu profonds}

L'équation (I) peut être utilisée également pour les sédiments afin de décrire l'équilibre de l'o-P dans l'eau et le sédiment, en supposant :

a) un mélange complet des sédiments et de l'eau surnageante

b) la même valeur de B de l'isotherme (I), trouvée pour le $\mathrm{Fe}(\mathrm{OOH})$, appliquée aux sédiments.

L'équation devient ensuite :

$P_{\text {in }}=A * \sqrt[3]{(0-P)}+P_{\text {out }}+\Delta(0-P)$

où $\quad P_{\text {in }}=$ phosphate entrant dans le lac $\left(\mathrm{g} \mathrm{m}^{-2} \mathrm{a}^{-1}\right)$ $P_{\text {out }}=$ phosphate sortant du lac $\left(\mathrm{g} \mathrm{m}^{-2} \mathrm{a}^{-1}\right)$ $\Delta(\mathrm{o}-\mathrm{P})=$ Changement de la concentration du phosphate dans l'eau ( $\left.\mathrm{g} \mathrm{m}^{-2} \mathrm{a}^{-1}\right)$

Les valeurs de $B$ pour les sédiments ne sont pas toujours exactement 0.33 , mais elles en diffèrent peu, par exemple 0.31 - 0.45 (Voir Fig. 1). Quelle que soit cette valeur, le principe du raisonnement qui suit reste le même, mais numériquement la solution devient difficile.

En remplaçant dans l'équation (VI) $\left(P_{\text {in }}-P_{\text {out }}\right)$ par $n^{*} L$ (charge nette accumulée), nous pouvons écrire pour la $\mathrm{n}^{\text {ième année : }}$

$$
\begin{aligned}
\mathrm{n}^{*} \mathrm{~L} & =\left(\mathrm{P}_{\text {sed }}\right)_{\mathrm{n}}+(\mathrm{o}-\mathrm{P})_{\mathrm{n}} \\
\mathrm{ou} & \\
\mathrm{n}^{*} \mathrm{~L} & =\mathrm{A}^{*} \sqrt[3]{(0-\mathrm{P})_{\mathrm{n}}}+(\mathrm{o}-\mathrm{P})_{\mathrm{n}}
\end{aligned}
$$

Cette équation n'a qu'une valeur racine réelle, qui nous permet de calculer $P_{\text {sed }}$ et o-P en fonction de $n^{*} L$; c'est-à-dire en fonction du temps. Les figures $5 a$ et $5 b$ représentent ce calcul de $P_{\text {sed }}$ et o-P. Dans un premier temps, on remarque que l'o-P est presque entièrement fixé dans les sédiments : la concentration dans l'eau reste faible, ensuite, elle augmente plus rapidement. Ceci ne signifie pas que l'on ait affaire à un autre processus mais s'explique par le fait que le mécanisme d'adsorption soit modélisé par une racine cubique. Sur cette figure, nous avons également décrit ce qui se produisait si la charge externe était interrompue (flèches verticales). Deux cas sont considérés : le cas d'un lac à taux de renouvellement annuel et celui d'un lac ne se renouvelant pas, comme les marais dans les zones humides méditerranéennes. La concentration en o-P va diminuer 


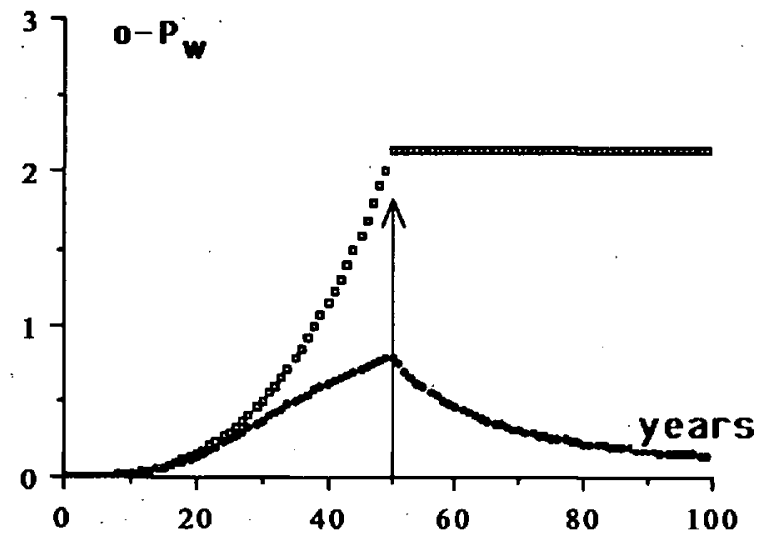

Fig. 5a. Evolution de l'ortho phosphate dissous dans un lac peu profond avec un taux de renouvellement de $1 \mathrm{an}^{-1}$ (courbe inférieure) et sans renouvellement (courbe supérieure). (Axe Y : $\mathrm{g} \mathrm{m}^{-2}$ de P). La flèche indique le moment où la charge en phosphate a été arrêtée.

Fig. 5a. Changes over time of $o-P_{w}$ in a shallow mud/water system with a flushing rate of $1 \mathrm{y}^{-1}$ (lower curve) and without flushing (upper curve). (Y axis : $\mathrm{g} \mathrm{m}^{-2}$ of $\mathrm{P}$ ). The arrow indicates the moment the $P$ input became 0 .

dans le lac avec renouvellement, mais très lentement. Le temps pour atteindre à nouveau la concentration d'origine sera plus long que la durée du chargement (stockage dans les sédiments). La restauration des lacs, et en particulier dés.lacs peu profonds, est un processus de longue haleine:: Les résultats escomptés d'un arrêt des apports peuvent être largement retardés.

Nous avons considéré ci-dessus que tous les phosphates entrant dans un écosystème aquatique étaient échangeables ; ceci n'est en fait pas le cas. La connaissance de la fraction organique et de son taux de minéralisation reste une préoccupation essentielle pour permettre une modélisation complète de ce processus.

\section{Conclusion}

Nous avons mis au point un schéma d'extraction des phosphates nous permettant de distinguer deux fractions minérales, $\mathrm{Fe}(\mathrm{OOH}) \approx \mathrm{P}$ et $\mathrm{CaCO}_{3} \approx \mathrm{P}$, ainsi que deux fractions organiques. La première fraction organique est extraite avec un acide fort et contient probablement des composés humiques. La deuxième, que l'on peut extraire avec du $\mathrm{NaOH}$, comporte essentiellement de l'hexaphosphate

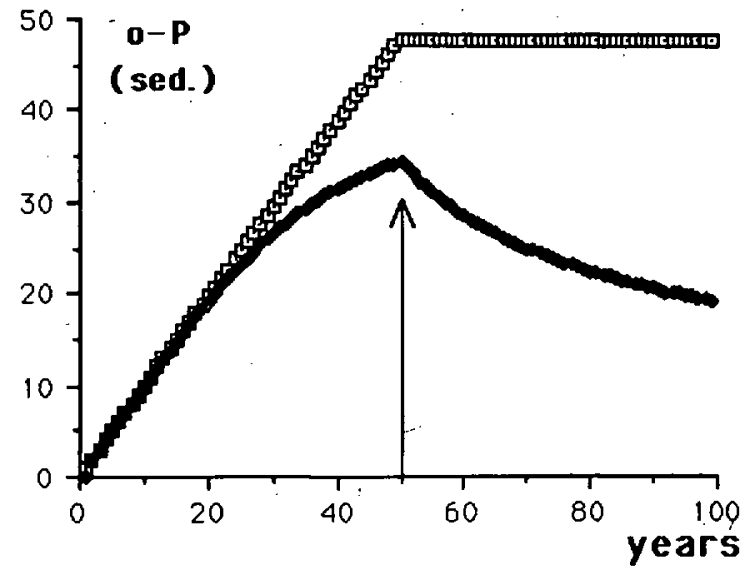

Fig. 5b. Evolution du phosphate adsorbé sur le sédiment dans un lac peu profond avec un taux de renouvellement de 1 an $^{-1}$ (courbe inférieure) et sans renouvellement (courbe supérieure). (Axe $\mathrm{Y}: \mathrm{g} \mathrm{m}^{-2}$ de P). La flèche indique le moment où la charge en phosphate a été arrêtée.

Fig. Sb. Changes over time of o- $P_{\text {sed }}$ in a shallow mud/water system with a flushing rate of $1 \mathrm{y}^{-1}$ (lower curve) and without flushing (upper curve). ( $\mathrm{Y}$ axis : $\mathrm{g} \mathrm{m}^{-2}$ of $\mathrm{P}$ ). The arrow indicates the moment the $P$ input became 0 .

d'inositol. La composition des phosphates inorganiques peut expliquer leur nò-biodisponibilité. Seuls les phosphates minéraux sont disponibles pour la croissance du phytoplancton.

Nous avons établi un diagramme de solubilité pour le $\mathrm{Fe}(\mathrm{OOH}) \approx \mathrm{P}$ et le $\mathrm{CaCO}_{3} \approx \mathrm{P}$ et montré que l'adsorption des o-P sur le $\mathrm{Fe}(\mathrm{OOH})$ était accrue en présence d'autres cations, naturellement présents dans l'eau douce.

Nous avons développé un modèle décrivant l'accumulation du phosphate dans le sédiment, et le relargage dans le cas où des mesures de restauration seraient entreprises.

Nous pensons que le FeS joue un rôle important dans le cycle des sels nutritifs. D'une part, la conversion de $\mathrm{FeOOH}$ en $\mathrm{FeS}$ diminue l'adsorption de l'o-P, d'autre part, le FeS intervient dans le processus de dénitrification (Fig. 6). Dans les sols recouverts d'eau (« water logged soils »), l'oxydation du FeS est plus facile que l'oxydation de la matière organique ; on peut donc améliorer la production végétale, naturelle ou agricole dans le cas du riz, en les asséchant périodiquement. Nous avons effectué un suivi de la composition chimique des sédiments lors de l'assèchement d'un marais. Des changements 


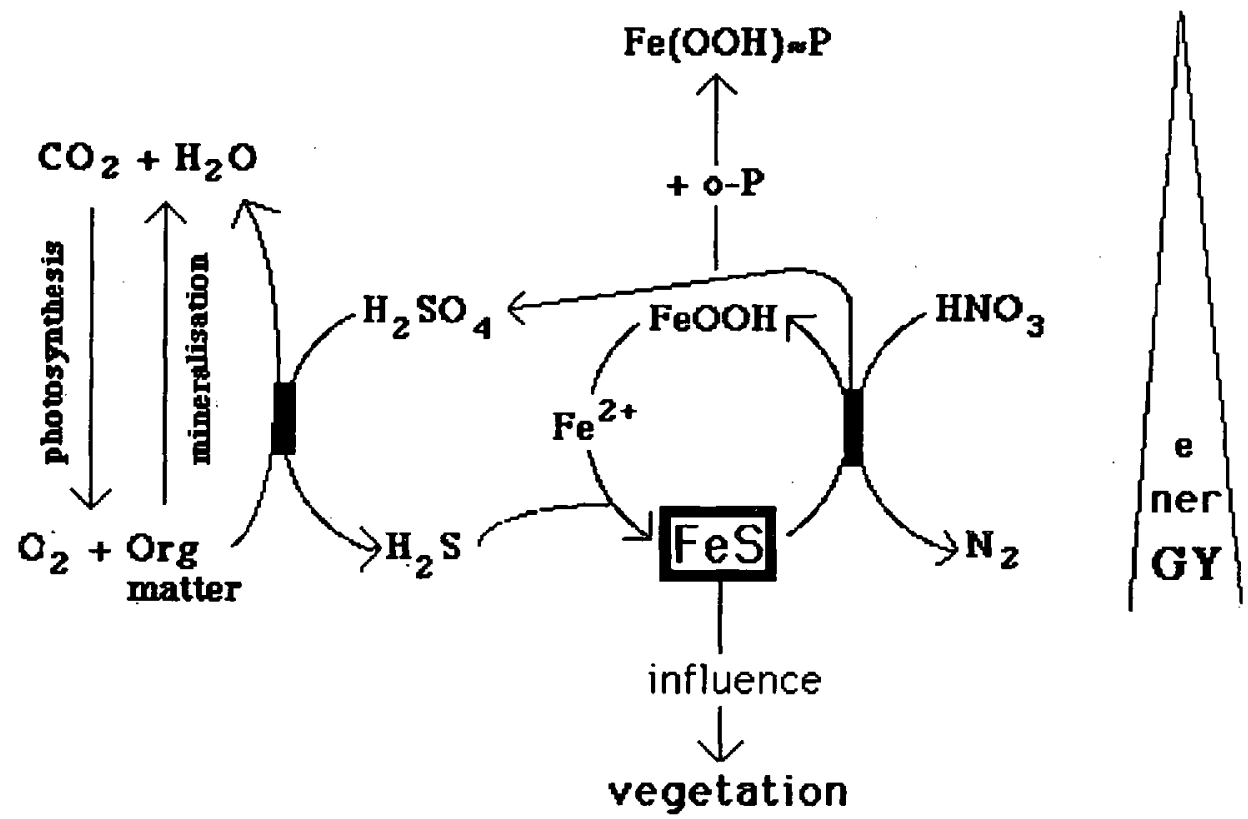

Fig. 6. Les relations entre photosynthèse, dénitrification, réduction de sulfate et adsorption de phosphate aux sédiments.

Fig. 6. Relations between photosynthesis, dénitrification, sulfate reduction and phosphate binding onto sediments.

importants apparaissent et permettent de proposer une meilleure gestion des eaux peu profondes.

L'accumulation du phosphate dans les sédiments a une grande influence sur l'eutrophisation des zones humides, où, en plus, le cycle de l'azote doit être pris en compte. Sur ce sujet, Golterman (1992, 1994b) a publié deux revues pour les zones humides méditerranéennes.

\section{Remerciements}

Nous tenons à remercier M. A. Booman pour les analyses au laboratoire, Me J.C. Golterman-Hardenberg, MM. A. Fabre et J. Bontoux pour leur assistance dans la préparation du manuscrit, le Sretie (contrats 89215 et 89302 ) et la C.E.C. (contrat EV4V - 0134-F) pour l'assistance financière.

\section{Travaux cités}

Arvieu J.-C. 1981، - Réactions des phosphates minéraux en milieu calcaire ; conséquences sur.l'état et la solubilité du phosphore. Bulletin de l'Association pour l'étude du sol : « Science du Sol », XX : 179-190.

Barrow N.J. 1985. - Reaction of anions and cations with variable-charge soils. Advances in Agronomy, 38 : 183-231.
Batsula A.A. \& Krivonosova G.M. 1973. - Phosphorus in the Humic and Fulvic acids of some Ukranian soils. Soviet Soil Sci : 347-350.

Buffle J. 1988. - Complexation Reactions in Aquatic Systems. E. Horwood Ltd. Chichester. 692 p.

Clymo R.S. \& Golterman H.L. 1985. - Precision and accuracy of the determination of the ionic product of hydroxy-apatite. Hydrobiologia, 126 : 31-34.

De Graaf Bierbrauwer-Würtz I.M. \& Golterman H.L. 1989. Fosfaatfracties in de bodem van een aantal Nederlandse meren. (in Dutch, with English summary). $\mathrm{H}_{2} \mathrm{O}, 22$ : 411-414.

De Groot C.J. 1990. - Some remarks on the presence of organic phosphates in sediments. Hydrobiologia, 207 : 303-309.

De Groot C.J. 1991. - The influence of FeS on the inorganic phosphate system in sediments. Verh. Int. Ver. Limnol., 24 : 3029-3035.

De Groot C.J. \& Fabre A.C. 1993. - The impact of desiccation of a freshwater marsh (Garcines Nord, Camargue, France) on the sediment-water-vegetation interactions. Part $3:$ The fractional composition and the phosphate adsorption characteristics of the sediment. Hydrobiologia, 252 : 105-116.

De Groot C.J. \& Golterman H.L. 1989. - Gefractioneerde extracties van bodemfosfaten. $\mathrm{H}_{2} \mathrm{O}, 22: 66-67$ (in Dutch with English summary).

De Groot C.J. \& Golterman H.L. 1990. - Sequential fractionation of sediment phosphate. Hydrobiologia, 192 : 143-148. 
De Groot C.J. \& Golterman H.L. 1993. - On the presence of organic phosphate in some Camargue sediments : evidence for the importance of phytate. Hydrobiologia, 252, 117-126.

Fox L.E. 1993. - The chemistry of aquatic phosphate : inorganic processes in rivers. Hydrobiologia, $254: 1-16$.

Francko D.A. \& Heath R.T. 1983. - Abiotic uptake and photodependent release of phosphate from high-molecular-weight Humic Iron Complexes in Bog lakes. In : Christman R.F. \& E.T. Gjessing (Eds), Aquatic and Terrestrial Humic Materials. Ann Arbor Science Publ,, Ann Arbor, p. 467-481.

Frimmel F.H. \& Christman R.F. (Eds). 1988. - Humic substances and their role in the environment. Wiley \& Sons. Chichester, $270 \mathrm{p}$.

Ghosh A.K., Ghosh J.C. \& Prasad B. 1980. - Third Dissociation Constant of Phosphoric Acid from 283.15 K to 323.15 K J. Indian Chem. Soc., 57 : 1194-1199.

Gjessing E.T. 1976. - Physical and chemical characteristics of Aquatic Humus. Ann Arbor Science Publ. Ann Arbor. 120 p.

Goldberg S. \& Sposito G. 1984. - A chemical model of phosphate adsorption by soils. Soil Soc. Am. J. : $48:-772-778$.

Golterman H.L. 1982. - Differential extraction of sediment phosphate with NTA solutions. Hydrobiologia, $91: 683-687$.

Golterman H.L. 1984. - Sediments, modifying and equilibrating factors in the chemistry of freshwaters. Verh. Int. Ver. Limnol.; 22 : 23-59.

Golterman H.L. 1988a. - The calcium and iron bound phosphate phase diagram. Hydrobiologia, $159:$ 149-151.

Golterman H.L. 1988b. - Reflections on fractionation and bioavailability of sediment bound phosphate. Arch. Hydrobiol. Beih. Ergebn. Limnol. $30: 1-4$.

Golterman H.L. 1992. - Wetlands as nutrient buffers between continental and marine waters. Bull. de l'Institut Océanographique, Numéro spécial 11 : 75-87.

Golterman H.L. 1994a. - The role of the ferric hydroxidephosphate-sulphide system in sediment water exchange. Hydrobiologia, sous presse.

Golterman H.L. 1994b. - The labyrinth of nutrient cycles and buffers in wetlands ; Results based on research in the Camargue (Southern France). Hydrobiologia, sous presse.

Golterman H.L. \& Booman A. 1988. - Sequential extraction of iron-phospate and calcium- phosphate by chelating agents. Verh. Int. Ver. Limnol., 23 : 904-909.

Golterman H.L. \& Meyer M.L. 1985. - The geochemistry of two hard water rivers, the Rhine and the Rhone. Part 4 : The determination of the solubility product of hydroxy-apatite. Hydrobiologia. $126: 21-24$.

Golterman H.L. \& de Oude N.T. 1991. - Eutrophication of Lakes, Rivers and Coastal Seas. In : Hutzinger O. (Ed.), The Handbook of Environmental Chemistry, Vol. 5A, Springer Verlag, Berlin, Heidelberg : 79-124.

Grobbelaar J.U. 1983. - Availability to algae of N and P adsorbed on suspended solids in turbid waters of the Amazon River. Arch. Hydrobiol., $96: 302-316$.

Grobler D.C. \& Davies E. 1979. - The availability of sediment phosphate to algae. Water $S A, 5: 114-123$.

Grobler D.C. \& Davies E. 1981 . - Sediments as a source of phosphate ; A study of 38 impoundments. Water $S A, 7$ : 54-60.
Kuo S. \& Lotse E.G. 1974. - Kinetics of phosphate adsorption and desorption by lake sediments. Soil Sci. Soc. Amer. Proc., $38: 50-54$.

Lijklema L. 1977. - The role of iron in the exchange of phosphate between water and sediment. In : Golterman H.L. (Ed.), Interactions between Sediments and Freshwater, Junk, the Hague : 313-317.

Lindsay W.L. 1989. - Chemical equilibria in soils. Wiley, New York, $449 \mathrm{p}$.

Mortimer C.H. 1941. - The exchange of dissolved substances between mud and water in lakes. J. Ecol., 29 : 280-329.

Mortimer C.H. 1942. - The exchange of dissolved substances between mud and water in lakes. J. Ecol., $30: 147-201$.

Moutin Th. 1992. - Contribution à l'Etude du Cycle du Phosphate dans les Ecosystèmes lagunaires. Thèse Université de Montpellier II, Sciences et techniques du Languedoc.

López P. \& Morgui J.A. 1992. - Phosphate and calcium carbonate saturation in a stratified coastal lagoon. Hydrobiologia, $228: 55-63$.

Olsen S. 1964. - Phosphate equilibrium between reduced sediments and water, laboratory experiments. with radioactive phosphorus. Verh. Int. Ver. Limnol., 13 : 915-922.

Parfit R.L., Russel J.D. \& Farmer V.C. 1976. - Confirmation of the surface structures of goethite $(\alpha-\mathrm{FeOOH})$ and phosphated goethite by infrared spectroscopy. I. Chem. Soc. Farad. Trans. 72 : 1082-1087.

Staudinger B., Peiffer S., Avnimelech Y. \& Berman T. 1990. Phosphorus mobility in interstitial waters of sediments in Lake Kinneret, Israel. Hydrobiologia, 207 : 167-177.

Stumm W., Kummert R. \& Sigg L. 1980. - A ligand exchange model for the adsorption of inorganic and organic ligands at hydrous oxide interfaces. Croat. Chem. Acta, $53: 291-312$.

Sposito G. 1984. - The surface chemistry of soils. Oxford Clarendon Press. 234 p.

Sposito G. 1989. - The chemistry of soils. Oxford University Press. 277 p.

Sverjensky D.A. 1993. - Physical surface-complexation models for sorption at the mineral-water interface. Nature, 364 : 776-780.

Syers J.K. \& Curtin D. 1989. - Inorganic reactions controlling prosphorus cycling. In Thiessen H. (Ed), Phosphorus cycles in terrestrial and Aquatic ecosystems. Proceeding of a SCOPE workshop, May 1988, Czerniejewo, Poland. Saskatchewan Institute of Pedology, Saskatoon, Canada.

Tipping E. 1985. - Accumulation of humic substances in the hypolimnion of a seasonally anoxic lake following cosedimentation with iron oxide. Org. Geochem., $8: 123-124$.

Tipping E. \& Cooke D. 1982. - The effects of adsorbed humic substances on the surface charge of goethite $(\alpha-\mathrm{FeOOH})$ in freshwaters. Geochimica et Cosmochimica Acta, 46 : 75-80.

Tipping E. \& Woof C. 1983. - Elevated concentrations of humic substances in a seasonally anoxic hypolimnion : evidence for co-accumulation with iron. Arch. Hydrobiol., 98 : 137-145. 